\title{
Measuring Hedge Effectiveness for FAS 133 Compliance
}

\author{
John M. Charnes* \\ The University of Kansas \\ School of Business \\ Lawrence, KS 66045 \\ jmc@ku.edu
}

\author{
Henk Berkman \\ School of Commerce \\ University of Auckland \\ Auckland, New Zealand \\ h.berkman@auckland.ac.nz
}

\author{
Paul Koch \\ The University of Kansas \\ School of Business \\ Lawrence, KS 66045 \\ pkoch@ku.edu
}

\begin{abstract}
Financial Accounting Standard (FAS) 133 requires business entities to document their anticipation of hedge effectiveness in order to qualify for hedge accounting treatment of gains and losses from financial derivatives. Meaningful assessment of anticipated hedge effectiveness must consider two distinct aspects of a firm's hedging strategy: (i) the strength of the hedging relation, which depends on the correlation between price changes in the hedged item and the hedging instrument under consideration; and (ii) the position taken in the hedging instrument relative to the optimal position. We propose an operational definition of hedge effectiveness that addresses both aspects of the hedging strategies under consideration. We then develop alternative measures of hedge effectiveness that take into consideration both the strength of the hedging relation and the hedge actually employed. This approach enables the user to evaluate the relative merits of alternative hedging strategies and thereby support risk management decisions, and to document the anticipation that a selected hedging strategy will be effective for compliance with FAS 133.

Key Words: Derivatives, Risk Management, Hedge Accounting, FAS 133
\end{abstract}

\section{Introduction}

Financial Accounting Standard (FAS) No. 133 requires changes in the market value of derivatives to be reported as earnings in the current period, unless they are used effectively to hedge specific risks faced by the firm. If an effective hedging relation is documented, the firm may qualify for hedge accounting treatment, whereby gains or losses on the hedging instrument are recognized in the same period as the income effects of the underlying hedged item. ${ }^{1}$

${ }^{*}$ Preliminary-Please do not quote without permission. The authors gratefully acknowledge the contributions to this work of the following individuals: Dave Fredericks, Ira Kawaller, Paul Munter, Kathleen Murphy, Vern Richardson, Sue Scholz, and Matt Wojewuczki.

${ }^{1}$ In accordance with the language of FAS 133, we refer to the asset or exposure responsible for the risk under consideration as the hedged item, the derivative asset used to offset that risk as the hedging instrument, and the interaction be-
It can be critical for businesses that use derivatives for risk management to qualify for hedge accounting treatment. Failure to qualify can have considerable tax consequences. Furthermore, without hedge accounting the mismatch in the timing of income recognition may induce income volatility that does not accurately reflect the underlying economics of the hedging relation. This income volatility can have a substantial impact on other managerial decisions and contractual obligations faced by the firm, and might influence the choice of the hedging instrument or even whether to hedge at all. ${ }^{2}$

Under FAS 133, businesses that hedge with derivatives can qualify for hedge accounting treatment only if they document, in advance, the anticipation that their hedging strategy will be "highly effective." An assessment of hedge effectiveness is required by FAS 133 at least every three months and whenever financial statements or earnings are reported by the firm. However, while the Financial Accounting Standards Board (FASB) stipulates the need to document this anticipation, it leaves the choice of the supporting methodology to the discretion of the hedger. The FASB does refer to the possible use of regression or correlation analysis to support the anticipation of hedge effectiveness, but without providing specific guidelines for applying

tween their price changes as the hedging relation.

${ }^{2}$ An extreme example of the consequences of income volatility resulting from failure to qualify for hedge accounting is the case of Franklin Savings and Loan. In 1990 Franklin experienced losses on a hedging instrument they claimed would be offset by subsequent expected gains in their business. They documented their anticipation of hedge effectiveness using a novel method to measure the strength of the hedging relation. In addition, they volunteered that without hedge accounting treatment, the resulting income statement volatility could trigger debt covenants that might further reduce the firm's equity below minimum capitalization requirements. This hedge accounting issue led regulators to close the savings and loan, ultimately resulting in its demise. See Koch (1995, p. 308) for further discussion of this case. 
these methods or specifying what minimal standards must be met to qualify for hedge accounting treatment.

In the absence of specific guidelines, the industry has come to embrace the "80-125 dollar offset ratio standard" as a widely used reference for effectiveness testing. The dollar offset ratio is defined as the change in the value of the hedging instrument divided by the change in the value of the hedged item over the assessment period or hedge horizon (PriceWaterhouseCoopers 1999). Under this standard a hedge qualifies as effective if there is a high degree of confidence that the dollar offset ratio expressed as a percentage will remain within the $80 \%-125 \%$ band over the hedge horizon.

Prior studies discuss various problems regarding compliance with the FAS 133 requirement for measuring anticipated hedge effectiveness. For example, Canabarro (1999) shows that under reasonable conditions for the processes generating prices of the hedged item and the hedging instrument, the dollar offset ratio gives false signals regarding hedge effectiveness. He demonstrates that this ratio is likely to fall frequently outside the $80 \%-125 \%$ band, even when the prices of the hedged item and the hedging instrument are highly correlated. Along another vein, Kawaller and Koch (2000) discuss analytical and practical problems with regression analysis as a tool for effectiveness testing using historical data. They clarify the proper use of regression methodology to measure the effectiveness of a hedging relation, and they suggest several alternative, non-regression measures for assessing hedge effectiveness to comply with FAS 133 . Their proposed measures focus on the variance of the combined hedged position (the portfolio composed of the hedged item and the amount of the hedging instrument selected by the user) relative to the variance of the unhedged position (the hedged item alone). Kalotay and Abreo (2001) propose an alternative measure of hedge effectiveness in the same spirit as the measures proposed by Kawaller and Koch (2000). They further suggest that users combine a backward-looking measure using historical data with an analogous forward-looking measure generated from bootstrapping simulation.

This manuscript contributes to the dialogue by outlining a basic framework for assessing anticipated hedge effectiveness. This framework is based on a two-part operational definition of hedge effectiveness that distinguishes between measuring the anticipated: (i) potential effectiveness of a hedging relation, and (ii) attained effectiveness of a selected hedged position.
The first part of this definition, potential effectiveness of a hedging relation, refers to the strength of the hedging relation and the amount of risk reduction possible by applying the optimal (minimum risk) hedge ratio to a given hedging instrument. The strength of the hedging relation depends upon the correlation between price changes in the hedged item and the hedging instrument under consideration. The amount of risk reduction possible likewise depends upon the square of this correlation. Correlation or regression analysis should thus reveal the strength of the hedging relation for various alternative hedging instruments, and thereby aid in the choice of the preferred hedging instrument. This analysis also reveals the extent of risk reduction possible, given the choice of the preferred hedging instrument and the optimal hedge ratio.

The second part of this definition, attained effectiveness of a selected hedged position, refers to the extent of risk reduction attained by the user's choice of both the hedging instrument and the hedge ratio (i.e., the position actually taken in the hedging instrument relative to the hedged item). This attained effectiveness can be measured by assessing the volatility of the combined hedged position, given the hedging instrument and hedge ratio actually chosen, relative to the volatility of the unhedged position (i.e., the hedged item by itself).

Our two-part definition provides a framework that makes a clear distinction between hedging and speculation, to assess hedge effectiveness and document compliance with FAS 133. This framework also leads us to propose several alternative hedge effectiveness measures that gauge the extent of risk reduction achieved by the user. These measures account for both the choice of the hedging instrument and the amount of this hedging instrument actually held.

The paper proceeds as follows. Section 2 presents our framework for distinguishing between hedging and speculation, in terms of the variance of the combined hedged position relative to the variance of the unhedged position. With our proposed framework as a background, Section 3 discusses drawbacks of the 80-125 dollar offset ratio standard and Section 4 reviews other previously suggested statistical methods for measuring anticipated hedge effectiveness. Section 5 provides our recommended alternative hedge effectiveness measures. A final section concludes.

Risk managers need clear guidance in finding an appropriate way to measure hedge effectiveness, in how to apply statistical methods properly to generate these effectiveness measures, and in how to in- 
terpret the results of their analyses. Without clear understanding of these statistical analyses or the expectations of the regulators for compliance with FAS 133, managers may be motivated to choose sub-optimal hedging strategies or even not hedge at all. $^{3}$ The Appendices discuss related issues for further inquiry and provide specific statistical methods for applying these procedures.

\section{Hedging versus Speculation}

\subsection{The Framework}

One of the difficulties with measuring hedge effectiveness is that 'the word 'hedge' is so ill defined and flexible that virtually any transaction can be characterized as a hedge" (Linsmeier and Pearson, 2000). Because it is impossible to measure that which is undefined, we present an operational definition of hedging that provides a framework to assess hedge effectiveness.

Let $S_{t}$ denote the unit price of the hedged item and $F_{t}$ denote the unit price of the hedging instrument at time $t$. The hedge ratio, $h$, is defined as the amount of the hedging instrument to be sold (bought) for every unit of the hedged item to be held long (short). A hedging strategy involves choosing a hedging instrument and an appropriate hedge ratio to accomplish the risk management objectives of the user. The unit value of the user's combined hedged position at time $t$ is then $C_{t}=S_{t}-h F_{t}{ }^{4}$

The change in the unit value of the combined hedged position from time $t-1$ to time $t$ is $\Delta C_{t}=$ $\Delta S_{t}-h \Delta F_{t}$, where $\Delta C_{t}=C_{t}-C_{t-1}, \Delta S_{t}=$ $S_{t}-S_{t-1}$, and $\Delta F_{t}=F_{t}-F_{t-1}$. Ideally, the change in value should be measured over a period of time equivalent to the user's hedge horizon. In practice, use of quarterly time periods is a feasible way to document anticipated hedge effectiveness and comply with FAS 133 (see Kawaller and Koch 2000).

The risk associated with the hedging strategy is the amount of uncertainty about future changes in the value of the combined hedged position. For a linear hedge, this uncertainty is related to the variance of the combined hedged position, which is

$$
V_{C}=V_{S}+h^{2} V_{F}-2 \rho h \sqrt{V_{S} V_{F}},
$$

\footnotetext{
${ }^{3}$ See Haushalter (2001) for a discussion of economic rationale for hedging.

${ }^{4}$ This framework applies only to linear hedges (i.e., those using forwards or futures contracts). Non-linear hedges (i.e., those using options) are beyond the scope of this study.
}

where $V_{S}=\operatorname{Var}\left(\Delta S_{t}\right), V_{F}=\operatorname{Var}\left(\Delta F_{t}\right)$, and $\rho=$ $\operatorname{Corr}\left(\Delta S_{t}, \Delta F_{t}\right)$. For a given hedged item, the choice of hedging instrument then determines the values of $V_{F}$ and $\rho$ in (1). ${ }^{5}$

Assuming the existence of a hedging relation (i.e., $\rho \neq 0$ ), choice of an appropriate hedge ratio, $h$, enables the hedger to create a combined position having smaller variance $\left(V_{C}\right)$ than that associated with the underlying hedged item alone $\left(V_{S}\right)$. The user may select any hedge ratio $(h)$ desired to manage risk. A hedger will choose a futures position that reduces risk while a speculator will choose a futures position that increases risk.

In Figure 1 we plot $V_{C}$ versus $h$ for selected values of $\rho, V_{F}$, and $V_{S}$ to illustrate how the variance of the combined position behaves as a function of the hedge ratio. Note that this function is a parabola with minimum variance attainable:

$$
V_{C}^{\star}=\left(1-\rho^{2}\right) V_{S} .
$$

This minimum risk attainable occurs at the optimal hedge ratio,

$$
h^{\star}=\rho \sqrt{V_{S} / V_{F}}
$$

(see Ederington (1979), Hull (2002, p.84) and Appendix A for details).

It is the prerogative of the user to determine the extent of the risk exposure $\left(V_{C}\right)$ desired. Figure 1 shows that the user can employ a financial derivative as a hedging instrument to attain any level of risk below $V_{S}$, down to $V_{C}^{\star}$, by varying $h$ between 0 and $2 h^{\star}$. Of course, the user may also use the derivative to take on additional risk above $V_{S}$ by varying $h$ outside the bounds 0 to $2 h^{\star}$.

It is the responsibility of the user to document anticipated hedge effectiveness to qualify for hedge accounting treatment under FAS 133. Expressions (1)-(3), along with Figure 1, provide a simple framework to document: $(i)$ the strength of the hedging relation $(\rho)$ and the minimum risk attainable for a given hedging instrument $\left(V_{C}^{\star}\right.$ attained at $\left.h^{\star}\right)$; and (ii) the level of risk actually attained with the chosen hedge ratio $\left(V_{C}\right.$ attained at $\left.h\right)$.

It is the responsibility of the regulators to determine whether the user is employing derivatives to increase or decrease risk. This determination calls for clear guidelines on acceptable methods for measuring and documenting whether a given position in a derivative represents hedging or speculation.

\footnotetext{
${ }^{5}$ See Ederington (1979) and Hull (2002, p. 84). Choice of the preferred hedging instrument should aim toward selecting a hedging instrument having the strongest possible hedging relation with the hedged item (i.e., the highest $\rho$ ), given other considerations germane to the hedger's problem such as liquidity and transactions costs.
} 


\subsection{Hedging versus Speculation}

In this framework we define hedging as taking a position in a hedging instrument (i.e., choosing $h$ ) such that the variance of the combined hedged position is less than the variance of the hedged item itself (i.e., $V_{C}<V_{S}$ ). Likewise, we define speculating as choosing a value of $h$ such that the variance of the combined position is greater than the variance of the unhedged position $\left(V_{C}>V_{S}\right)$.

Note again that for any choice of $h$ such that $0<h<2 h^{\star}$, the variance of the combined hedged position $\left(V_{C}\right)$ is less than the variance of the unhedged asset. Thus, any combined position that includes a relative amount of the hedging instrument within these bounds satisfies our definition of hedging and should be considered a bona fide hedge that qualifies for hedge accounting treatment under FAS 133.

It is clear from (2) that the potential effectiveness of a hedging strategy $\left(V_{C}^{\star}\right)$ in reducing the risk of the unhedged position $\left(V_{S}\right)$ depends only on the correlation between the hedged item and the hedging instrument $(\rho)$. However, expression (1) shows that the attained effectiveness of a selected hedging strategy $\left(V_{C}\right)$ depends upon three factors: $(i)$ the relative variances of the hedged item and the hedging instrument $\left(V_{S}\right.$ and $\left.V_{F}\right) ;(i i)$ the correlation between the hedged item and the hedging instrument $(\rho)$; and $($ iii $)$ the hedge ratio selected by the user $(h)$.

Analysis of $V_{S}, V_{F}$, and $\rho$ for alternative hedging instruments documents the potential effectiveness of alternative hedging relations, and allows choice of the hedging instrument that provides the maximum risk reduction possible (i.e., the lowest $V_{C}$ attainable). Given this choice of the hedging instrument, the hedge ratio chosen $(h)$ reflects the extent to which the user actually reduces risk toward the minimum risk attainable. A meaningful assessment of hedge effectiveness should account for both the potential effectiveness of the hedging relation and the attained effectiveness of the selected hedged position.

\section{$2.3 \quad$ Non-Optimal Hedging}

Our definition of hedging requires the user to choose a hedge ratio $(h)$ between 0 and $2 h^{\star}$, so that $V_{C}<V_{S}$. To qualify as hedging, we do not require the user to apply the optimal (minimum variance) hedge ratio, $h^{\star}$. While any hedge ratio other than $h^{\star}$ is non-optimal, hedge ratios between 0 and $2 h^{\star}$ still effectively reduce risk.
For example, FAS 133 stipulates that a futures hedge ratio of 1.0 may generally qualify as a bona fide hedge, and provides directions for hedge accounting given this choice of hedge ratio (see Kawaller and Koch 2000). In certain applications, a hedge ratio of 1.0 may constitute an appropriate hedge if the maturity of the hedging instrument selected matches the hedge horizon, and if the user intends to hold the derivative position until this maturity. However, as a practical matter, there is often no such perfect matching of maturities, and the user should be free to adjust the risk exposure ( $h$ and $V_{C}$ ) at any time. The resulting basis risk for the combined hedged position depends on the correlation between the hedged item and the hedging instrument, as well as the mismatch in their maturities. In this case, the minimum variance hedge ratio, $h^{\star}$, will typically deviate from one, according to $(3)$.

Consider the case in which $h^{\star}<1$, as in Figure 1. If the user varies the hedge ratio from zero toward $h^{\star}$, the user is clearly reducing risk. Any position in the hedging instrument between a hedge ratio of zero and $h^{\star}$ effectively hedges a portion of the total risk exposure embodied in the unhedged position, so that this use of derivatives should qualify for hedge accounting treatment under FAS 133.

Figure 1 demonstrates that if the user continues to increase the hedge ratio beyond $h^{\star}$ toward 1.0, the user is now increasing risk above the minimum risk attainable at $V_{C}^{\star}$. However, it would be inappropriate to disqualify such a choice of hedge ratio for hedge accounting treatment, given the stipulation in FAS 133 pertaining to a hedge ratio of 1.0. We further argue that, if the user continues to increase the hedge ratio above 1.0 toward $2 h^{\star}$, this use of derivatives should also qualify for hedge accounting treatment since it still results in a reduced level of risk $\left(V_{C}<V_{S}\right){ }^{6}$

In summary, for a derivative position to qualify for hedge accounting treatment, we focus on the effectiveness of a hedging strategy (i.e., choice of hedging instrument and hedge ratio) in reducing risk $\left(V_{C}\right)$ below that of the unhedged position $\left(V_{S}\right)$. For compliance with FAS 133, we do not distinguish between the hedge effectiveness of a smaller hedge ratio closer to zero, and the effectiveness of a larger hedge ratio closer to $2 h^{\star}$. As shown in Figure 1 , both cases may reduce $V_{C}$ only slightly below $V_{S}$. We believe that the FASB should allow hedge accounting treatment when the user has documented that the chosen hedging instrument and

\footnotetext{
${ }^{6}$ This problem becomes more complicated if $h^{\star}<0.5$, because then $2 h^{\star}<1.0$.
} 
the selected hedge ratio will result in a combined hedged position that has smaller variance than the unhedged position (i.e., $V_{C}<V_{S}$ ).

In Section 5 we propose alternative measures of hedge effectiveness that employ this framework to document both aspects of hedge effectiveness. However, we first discuss in Section 3 problems with the dollar offset ratio, then in Section 4 we summarize prior work on measuring hedge effectiveness.

\section{Dollar Offset Ratio}

One measure of hedge effectiveness that has received much attention is the dollar offset ratio, defined as:

$$
D_{t}=\frac{\Delta F_{t}}{\Delta S_{t}} .
$$

This ratio is intended to measure the ability of the hedging instrument to generate offsetting changes in the fair value or anticipated cash flows of the hedged item (PriceWaterhouseCoopers 1999). However, the dollar offset ratio does not explicitly consider either component of our two-part definition of hedge effectiveness: the strength of the hedging relation $(\rho)$, or the hedge ratio $(h)$ chosen by the user. Thus the dollar offset ratio does not appropriately measure the degree to which the hedger has effectively reduced risk.

Some FASB members support the notion that high effectiveness under FAS 133 should require that the dollar offset ratio for the chosen hedging instrument fall within a range of 0.80 to 1.25 for a large percentage of historical periods, while other FASB members believe the range should be 0.90 to 1.10 (PriceWaterhouseCoopers 1999). However, there is a technical difficulty in using the dollar offset ratio with either set of guidelines to measure hedge effectiveness.

Assume $S_{t}$ and $F_{t}$ follow Gaussian random walks, i.e., $S_{t}=S_{t-1}+\epsilon_{t}^{S}$ and $F_{t}=F_{t-1}+\epsilon_{t}^{F}$, where $\epsilon_{t}^{S}$ and $\epsilon_{t}^{F}$ are iid sequences such that $\Delta S_{t}=\epsilon_{t}^{S} \sim$ $N\left(0, V_{S}\right)$ and $\Delta F_{t}=\epsilon_{t}^{F} \sim N\left(0, V_{F}\right)$. If $V_{S}=V_{F}$ and $\operatorname{Corr}\left(\epsilon_{t}^{S}, \epsilon_{t}^{F}\right)=\rho$, then the dollar offset ratio, $D_{t}$, follows a Cauchy probability distribution with parameters $\rho$ and $\sqrt{1-\rho^{2}}$. The cumulative distribution function (cdf) of this distribution is:

$$
\operatorname{Pr}\left(D_{t}<d\right)=\frac{1}{2}+\frac{1}{\pi} \tan ^{-1}\left(\frac{d-\rho}{\sqrt{1-\rho^{2}}}\right)
$$

for $-\infty<d<\infty$ (Canabarro 1999).

The so-called 80-125 test uses the dollar offset ratio to gauge hedge effectiveness by keeping track
Table 1: Probabilities of the dollar offset ratio, $D_{t}=\Delta F_{t} / \Delta S_{t}$, falling outside the intervals $[0.80,1.25]$ and $[0.90,1.10]$ when $F_{t}$ and $S_{t}$ follow correlated Gaussian random walks having $V_{F}=V_{S}$, and the correlation, $\rho$, with the listed values. Probabilities are computed from Expression (5).

\begin{tabular}{|ccc|}
\hline & $P\left(D_{t}<0.80\right.$ or & $P\left(D_{t}<0.90\right.$ or \\
$\rho$ & $\left.D_{t}>1.25\right)$ & $\left.D_{t}>1.10\right)$ \\
\hline \hline 1.00 & .000 & .000 \\
0.99 & .362 & .608 \\
0.98 & .468 & .706 \\
0.95 & .614 & .807 \\
0.90 & .713 & .863 \\
0.50 & .879 & .945 \\
0.00 & .930 & .968 \\
\hline
\end{tabular}

of the relative frequency with which $D_{t}$ falls outside the interval $[0.80,1.25]$ over time. According to the 80-125 test, a hedge will be deemed ineffective if the dollar offset ratio falls outside the $[0.80,1.25]$ range more frequently than some unspecified upper bound, such as $1 \%, 5 \%$, or $10 \%$ of the time. Table 1 lists the probabilities that the dollar offset ratio will fall outside the intervals $[0.80,1.25]$ and $[0.90,1.10]$ for various levels of $\rho$. This table shows that the dollar offset ratio will fail the $80-125$ test quite often, even when the hedging relation is strong (i.e., price changes in the hedged item and the hedging instrument are very highly correlated). ${ }^{7}$

The fundamental problem with the dollar offset ratio is that small changes in $S_{t}$ result in large values of $D_{t}$. Thus the dollar offset ratio test is problematic when there is a high likelihood that the price change, $\Delta S_{t}$, will be near zero. In fact, the ratio in (4) is undefined if the values of $S_{t}$ and $S_{t-1}$ are identical (i.e., $\Delta S_{t}=0$ ). Further, the dollar offset ratio does not take into consideration the relative amount, $h$, of the hedging instrument that is actually included in the combined position, $C_{t}$. At best this measure offers only an slight indication of how well the hedging instrument tends to track the hedged item.

We propose that a meaningful assessment of hedge effectiveness should consider both the correlation of the hedged item with the hedging instrument $(\rho)$, and the amount of the hedging instrument $(h)$ included in the combined position. In

\footnotetext{
${ }^{7}$ Note that Gaussian random walk models for $S_{t}$ and $F_{t}$ are used in this illustration only to allow for analytic computation of the probabilities in Table 1. Use of non-Gaussian probability distributions for $\epsilon_{t}^{S}$ and $\epsilon_{t}^{F}$ would result in different non-zero probabilities of failures for these tests, but these probabilities would likely be more difficult to compute.
} 
the next section we describe several measures of effectiveness that have been set forth in the literature, and discuss the relative merits of each. This discussion is followed by our proposed alternative measures that account for both $\rho$ and $h$.

\section{Existing Measures of Effec- tiveness}

\subsection{Ederington}

Ederington (1979) may have been the first to recommend a measure of hedge effectiveness. His proposed measure is

$$
E=1-\frac{V_{C}^{\star}}{V_{S}}
$$

where $V_{C}^{\star}$ is the minimum variance attainable by taking the optimal combined position $C_{t}^{\star}=S_{t}-$ $h^{\star} F_{t}$. Thus $E$ measures the relative reduction in variance gained by taking the optimal combined position $\left(h^{\star}\right)$ for a given hedging instrument. By substituting $V_{C}^{\star}=V_{S}\left(1-\rho^{2}\right)$ into (6), it can be seen that the Ederington measure, $E$, is equal to $\rho^{2}$ for the optimal combined position. ${ }^{8}$ Note that this measure documents the greatest degree of risk reduction attainable $\left(V_{C}^{\star}\right)$ if the optimal hedge ratio, $\left(h^{\star}\right)$, is chosen, and reflects the strength of the hedging relation $(\rho)$. However, this measure ignores the extent to which the user actually reduces risk toward the minimum attainable, because it does not account for the hedge ratio $(h)$ actually selected.

\subsection{Kawaller and Koch}

Kawaller and Koch (2000) propose a measure of hedge effectiveness that does account for the hedged position selected by the entity. Their measure is

$$
K=\frac{V_{C}}{V_{S}} .
$$

Because $V_{C}$ represents variation in the combined hedged position that remains after the entity selects $h, V_{C}$ is analogous to the sum of squared residuals (SSE) in a regression model. Similarly, $V_{S}$ represents the total variation in the hedged item, and is

\footnotetext{
${ }^{8}$ The measure $E$ can be estimated easily for a chosen hedging instrument by finding the $R^{2}$ of a simple (unconstrained) regression of $\Delta S_{t}$ on $\Delta F_{t}$. That is $E=1-$ $V_{C}^{\star} / V_{S} \approx 1-\mathrm{SSE} / \mathrm{SST}=R^{2}$, where $\mathrm{SSE}$ is the sum of squared residuals and SST is the total sum of squares in the regression model. This measure is also discussed by Hull (2002, p. 85).
}

analagous to the total sum of squares (SST) in a regression model. Therefore, $K$ can be interpreted as the proportion of total risk (variance) that remains after hedging. A smaller value of $K$ indicates a more effective hedge. Kawaller and Koch (2000) do not specify the threshold of acceptability for $K$ to qualify for hedge accounting treatment. However, according to our definition, any hedged position for which $K<1$ would be considered hedging rather than speculation, and should qualify for hedge accounting treatment. Similarly, our definition of speculation corresponds to values of $K>1$ (i.e., $V_{C}>V_{S}$ ).

\subsection{Kalotay and Abreo}

Kalotay and Abreo (2001) present a measure they call the "volatility reduction measure (VRM)," which is related to Kawaller and Koch's measure $K$ and thus also accounts for the hedged position selected by the entity. This measure is

$$
\begin{aligned}
\mathrm{VRM} & =1-\frac{\sigma_{C}}{\sigma_{S}} \\
& =1-\sqrt{K},
\end{aligned}
$$

where $\sigma_{C}=\sqrt{V_{C}}$ and $\sigma_{S}=\sqrt{V_{S}}$ are the standard deviations of the combined position and the hedged item, respectively. Kalotay and Abreo list three reasons for using standard deviations in the computation of VRM: (i) they suggest that standard deviations are more meaningful to managers than variances; $(i i)$ they argue that this measure has a "common analytic framework with Value at Risk (VaR)" because both VRM and VaR use the standard deviation in their respective calculations; and (iii) they believe this measure is in accord with the $80-125$ rule, presumably as applied to the dollar offset ratio.

We agree that standard deviation may be a more meaningful statistic to some managers than variance because standard deviation is measured in the same units (dollars or cents) as the price changes in the hedged item and the hedging instrument. Further, many managers interpret standard deviations in terms of probabilities associated with returns, for example, as applied in Value at Risk. However, since the standard deviation is simply the square root of the variance, the two statistics are equivalent in their information content.

Note that Kawaller and Koch's (2000) measure, $K$, and Kalotay and Abreo's (2001) measure, VRM, do not distinguish between the strength of the hedging relation and the effectiveness of the hedge ratio employed. In practice, risk managers should assess 
each component of the hedging strategy, as well as overall hedge effectiveness.

\section{Measuring Hedge Effective- ness}

In this section we propose several measures of hedge effectiveness that compare attributes of the selected hedged position (given the selected hedge ratio, $h$ ) to the same attributes of the optimal combined position (with optimal hedge ratio, $h^{\star}$ ) that obtains minimum variance possible for the chosen hedging instrument. These respective measures assess: $(i)$ the potential effectiveness of a hedging instrument; (ii) the attained effectiveness of a selected hedge ratio; and (iii) the overall effectiveness that combines (i) and (ii).

\subsection{Hedging Instrument Effective- ness}

The first task in our proposed framework is to assess the potential effectiveness of a hedging instrument in reducing risk. This task calls for the measure $E$ proposed by Ederington (1979):

$$
\mathrm{HIE}=\frac{V_{S}-V_{C}^{\star}}{V_{S}}=E=\rho^{2} .
$$

This measures the potential effectiveness of the hedging relation if the optimal hedge ratio $\left(h^{\star}\right)$ is applied. It represents the maximum risk reduction possible $\left(V_{S}-V_{C}^{\star}\right)$ as a proportion of the total risk of the unhedged position $\left(V_{S}\right)$. In Figure 1, HIE is the vertical distance below $V_{S}$ that can be attained with the optimal hedge ratio $\left(h^{\star}\right)$, relative to the entire vertical distance depicting the total unhedged risk, $\left(V_{S}\right)$.

\subsection{Hedge Ratio Effectiveness}

The second task is to assess the attained effectiveness of a selected hedge ratio. Our second measure of hedge effectiveness therefore considers the extent of risk reduction attained with the selected hedge ratio $(h)$, relative to the maximum reduction in risk possible with $h^{\star}$ :

$$
\mathrm{HRE}=\frac{V_{S}-V_{C}}{V_{S}-V_{C}^{\star}}=\frac{V_{S}-V_{C}}{\rho^{2} V_{S}} .
$$

In Figure 1, HRE represents the vertical distance below $V_{S}$ attained with the chosen hedge ratio $(h)$ as a proportion of the vertical distance below $V_{S}$ attainable with the minimum variance hedge ratio $\left(h^{\star}\right)$. This measure reveals the extent to which the hedge ratio chosen reduces risk toward the minimum risk attainable. ${ }^{9}$ The maximum possible value of HRE is 1 , which occurs when the user chooses the minimum risk hedge ratio $\left(h=h^{\star}\right.$ and $V_{C}=V_{C}^{\star}$ ). Any combined position for which $0<\mathrm{HRE}<1$ will indicate hedging by our definition, while any negative values of HRE will indicate speculation. As $h$ is varied from zero to $h^{\star}$ to $2 h^{\star}$, HRE ranges from zero to one and back to zero. Thus, larger values of HRE up to 1.0 indicate more effective hedging. ${ }^{10}$

\subsection{Overall Hedge Effectiveness}

A third task is to combine information about the potential effectiveness of the hedging instrument (HIE) with the attained effectiveness of the hedge ratio chosen (HRE), into a measure of overall hedge effectiveness. In this regard, our third measure of overall hedge effectiveness is defined as the proportion of the unhedged item's total variance that is eliminated by taking the chosen combined position:

$$
\mathrm{OHE}=\frac{V_{S}-V_{C}}{V_{S}}=1-K=\mathrm{HIE} \times \mathrm{HRE} .
$$

In Figure 1, OHE represents the reduction in risk between the unhedged item and the combined hedged position $\left(V_{S}-V_{C}\right)$, relative to the total risk of the unhedged item $\left(V_{S}\right)$. Note that this overall effectiveness measure is simply the product of HIE and HRE. An increase in either factor will yield a larger measure of overall hedge effectiveness. Any combined position for which $0<\mathrm{OHE}<1$ will indicate hedging by our definition, while any position for which $\mathrm{OHE}<0$ will indicate speculation. Larger values of OHE indicate more effective hedging.

The procedure for applying any of these hedging effectiveness measures (HIE, HRE, or OHE) to document the anticipation of hedge effectiveness for compliance with FAS 133 is simply to document how the user obtains valid estimates of $V_{S}, V_{F}$, and $\rho$, and how these estimates are used to compute the respective hedge effectiveness measures. Appendix D offers a set of statistical techniques that

\footnotetext{
${ }^{9} \mathrm{HRE}$ suffers from the technical difficulty of division by zero when there is no hedging relation (i.e., $\rho=0$ ) because in that case $V_{C}^{\star}=V_{S}$ with $h^{\star}=0$.

${ }^{10} \mathrm{An}$ alternative means to measure hedge ratio effectiveness would be to take the ratio, $h / h^{\star}$. This approach measures the horizontal distance in Figure 1 attained with the chosen hedge ratio as a proportion of the minimum variance hedge ratio. See Appendix C for details.
} 
can be applied to obtain the necessary estimates for computing these measures of hedge effectiveness.

\section{Conclusion}

This study sets forth a two-part operational definition of hedge effectiveness. This definition leads to a simple framework for assessing anticipated hedge effectiveness, for risk management and compliance with FAS 133. Our framework makes a clear distinction between hedging and speculation on the part of the user. This framework is based on the proposition that meaningful assessment of anticipated hedge effectiveness for FAS 133 should consider both: $(i)$ strength of the hedging relation as determined by the choice of the hedging instrument, and (ii) the amount of the hedging instrument held in the combined hedged position. The measures of hedge effectiveness, HIE, HRE, and OHE proposed in this study account for both conditions. These hedge effectiveness measures have clear interpretations that reveal the extent to which the user effectively reduces risk, relative to the minimum risk attainable, and they can be used to distinguish between hedging and speculation.

\section{References}

[1] Canabarro, Eduardo, "A Note on the Assessment of Hedge Effectiveness Using the Dollar Offset Ratio Under FAS 133," Goldman Sachs research paper, June 1999.

[2] Ederington, L. H. (1979). "The hedging performance of the new futures markets," Journal of Finance, 34 (March), 157-170.

[3] FASB, "Statement of Financial Accounting Standards No. 133: Accounting for Derivative Instruments and Hedging Activities," Stamford, CT, 1998.

[4] Haushalter, David, "Why Hedge? Some evidence from oil and gas producers," Journal of Applied Corporate Finance, Vol. 14, No. 4 (Winter 2001), 87-92.

[5] Hull, J. C. (2002). Fundamentals of futures and options markets, Fourth edition. Upper Saddle River, NJ: Prentice-Hall.

[6] Kalotay, Andrew, and Leslie Abreo, "Testing Hedge Effectiveness for FAS 133: The volatility reduction measure," Journal of Applied Corporate Finance, Vol. 14, No. 4 (Winter 2001), 9399.

[7] Kawaller, Ira G., and Paul D. Koch, "Meeting the 'Highly Effective Expectation' Criterion for Hedge Accounting," Journal of Derivatives, 7 (Summer 2000), 79-87.

[8] Koch, Timothy W., (1995). Bank Management, Third edition. Orlando, FL: Dryden.

[9] Linsmeier, Thomas J., and Neil D. Pearson (2000). "Value at Risk," Financial Analysts Journal, (March/April), 47-67.

[10] PriceWaterhouseCoopers,

www.pwccomperio.com, Accounting and Reporting Manual (ARM), 1999.

\section{A Minimum Variance}

To determine the minimum variance attainable by the optimal hedged position, begin with expression (1), reproduced below as expression (A.1) for convenience:

$$
V_{C}=V_{S}+h^{2} V_{F}-2 \rho h \sqrt{V_{S} V_{F}} .
$$

Take the first derivative of (A.1) with respect to $h$ :

$$
\frac{d V_{C}}{d h}=2 h V_{F}-2 \rho \sqrt{V_{S} V_{F}} .
$$

Set (A.2) equal to zero and solve for $h$ to get the optimal hedge ratio:

$$
h^{\star}=\rho \sqrt{\frac{V_{S}}{V_{F}}} .
$$

Substitute $h^{\star}$ into (A.1) to get the minimum variance attained by the optimal combined hedged position:

$$
\begin{aligned}
V_{C}^{\star} & =V_{S}+\left(\rho \sqrt{\frac{V_{S}}{V_{F}}}\right)^{2} V_{F}-2 \rho\left(\rho \sqrt{\frac{V_{S}}{V_{F}}}\right) \sqrt{V_{S} V_{F}} \\
& =V_{S}+\rho^{2} V_{S}-2 \rho^{2} V_{S} \\
& =\left(1-\rho^{2}\right) V_{S} .
\end{aligned}
$$

\section{B Issues for Further Debate}

Note that a hedge ratio between one and $2 h^{\star}$ represents a reversal of the user's exposure from that in the unhedged item. For example, if the user takes a long position in the hedged item, this unhedged position will appreciate (depreciate) when 
$S_{t}$ rises (falls). A hedge ratio between zero and $h^{\star}$ then partially offsets the underlying movements in the hedged item, so that the combined position will appreciate (depreciate) less than the unhedged position when $S_{t}$ rises (falls). On the other hand, when $h$ is varied between one and $2 h^{\star}$ in Figure 1, the combined position will now appreciate (depreciate) following opposite movements in $S_{t}$ and $F_{t}$. That is, a hedge ratio between one and $2 h^{\star}$ now more than offsets the underlying movements in the hedged item, so that the combined position will now depreciate (appreciate when $S_{t}$ rises (falls). We maintain that such a hedge position should still qualify under FAS 133, as long as $V_{C}<V_{S}$.

We take this position regardless of the magnitude of the correlation, $\rho$, as long as this correlation is nonzero. Our proposed framework calls upon the user to document both the potential effectiveness of the hedging instrument selected, and the attained effectiveness of the actual hedge ratio selected. Our framework does not require some minimum value of the correlation coefficient before the hedging strategy qualifies for hedge accounting treatment. That is, to qualify for hedge accounting treatment, we do not distinguish between the validity of a hedging strategy in which the preferred hedging instrument demonstrates a high correlation with the hedged item, and another in which the hedging instrument reveals a low (but nonzero) correlation.

This view acknowledges that, for some risk exposures, the available hedging instruments may display hedging relations with relatively low correlations. We do not rule out a user's hedging strategy qualifying for hedge accounting, just because the 'best' hedging instrument available may fail to meet some ad hoc minimum correlation. While it is important for the user to analyze and document the extent of risk reduction possible, there is much room for debate regarding how low this attainable risk, $V_{C}^{\star}$, must be to qualify for hedge accounting (i.e., how high $\rho$ must be in relation to $V_{F}$ and $\left.V_{S}\right)$. We suggest the crucial test for qualifying under FAS 133 should instead involve documentation regarding both the extent of risk reduction possible with the user's choice of hedging instrument and whether the user has attained a lower level of risk $\left(V_{C}<V_{S}\right)$ with his or her choice of hedge ratio.

\section{Relative-to-optimal Hedge Ratio Effectiveness}

Another alternative measure of the effectiveness of the hedge ratio chosen is:

$$
\mathrm{RHRE}=\frac{h}{h^{\star}}=\frac{h}{\rho\left(\frac{\sigma_{S}}{\sigma_{F}}\right)}=\frac{h \sigma_{F}}{\rho \sigma_{S}} .
$$

In Figure 1, RHRE represents the ratio of the hedged position taken on the horizontal axis $(h)$ relative to the minimum variance hedge ratio $\left(h^{\star}\right)$. Any combined position for which $0<$ RHRE $<2$ will indicate hedging by our definition, while any other non-zero values of RHRE will indicate speculation. A combined position for which RHRE $=1.0$ is optimal in that it achieves minimum variance attainable for a given hedging instrument. ${ }^{11}$

The proximity of RHRE to 1.0 can be used as a measure of how close the selected hedge ratio is to the minimum variance hedge ratio. For example, it might be useful to apply some standard such as the $80-125$ (or 90-110) rule to this measure if one wishes to define effective hedging as choosing a hedge ratio that is within a specified percentage of the optimal position.

\section{Parameter Estimation}

Recommended estimation procedure for using HIE, HRE, OHE, and RHRE to document anticipated hedge effectiveness on a quarterly basis:

1. Obtain $T$ quarterly values or prices of the hedged item, $S_{t}$ and the hedging instrument, $F_{t}$ for $t=1, \ldots, T$. The number of past values, $T$, should be at least as large as the number of quarters for which the hedge will be held.

2. Find the mean changes of the hedged item,

$$
\widehat{\mu}_{S}=\frac{1}{T-1} \sum_{t=2}^{T}\left(S_{t}-S_{t-1}\right),
$$

and the mean changes of the hedging instrument,

$$
\widehat{\mu}_{F}=\frac{1}{T-1} \sum_{t=2}^{T}\left(F_{t}-F_{t-1}\right) .
$$

\footnotetext{
${ }^{11}$ RHRE suffers from the technical difficulty of division by zero when there is no hedging relation $(\rho=0)$ because in that case $h^{\star}=0$.
} 
3. Estimate the variance of the hedged item as:

$$
\widehat{V}_{S}=\frac{1}{T-2} \sum_{t=2}^{T}\left(S_{t}-S_{t-1}-\widehat{\mu}_{S}\right)^{2},
$$

and the variance of the hedging instrument as

$$
\widehat{V}_{F}=\frac{1}{T-2} \sum_{t=2}^{T}\left(F_{t}-F_{t-1}-\widehat{\mu}_{F}\right)^{2} .
$$

4. Estimate the covariance between the price changes in the hedged item and the hedging instrument as:

$$
\widehat{\operatorname{Cov}}_{S F}=\frac{1}{T-2} \sum_{t=2}^{T}\left(S_{t}-S_{t-1}-\widehat{\mu}_{S}\right)\left(F_{t}-F_{t-1}-\widehat{\mu}_{F}\right) .
$$

5. Estimate the correlation between the price changes in the hedged item and hedging instrument as:

$$
\hat{\rho}=\frac{\widehat{\operatorname{Cov}}_{S F}}{\sqrt{\widehat{V}_{F} \widehat{V}_{S}}} .
$$

If $\hat{\rho}=0$, there is no hedging relation, so don't hedge. If $\hat{\rho}=1$, the hedging relation is perfect, and $h^{\star}=\sqrt{V_{S} / V_{C}}$.

6. Using the selected hedge ratio, $h$, compute the estimate of the variance of the combined hedged position as:

$$
\widehat{V}_{C}=\widehat{V}_{S}+h^{2} \widehat{V}_{F}-2 \hat{\rho} h \sqrt{\widehat{V}_{S} \widehat{V}_{F}} .
$$

7. Calculate HIE as:

$$
\mathrm{HEI}=\hat{\rho}^{2} .
$$

8. Calculate HRE as:

$$
\mathrm{HRE}=\frac{\widehat{V}_{S}-\widehat{V}_{C}}{\hat{\rho}^{2} \widehat{V}_{S}} .
$$

9. Calculate $\mathrm{OHE}$ as:

$$
\mathrm{OHE}=\frac{\widehat{V}_{S}-\widehat{V}_{C}}{\widehat{V}_{S}} .
$$

10. Calculate RHRE as:

$$
\mathrm{RHRE}=\frac{h}{\hat{\rho}} \sqrt{\frac{\widehat{V}_{F}}{\widehat{V}_{S}}} .
$$




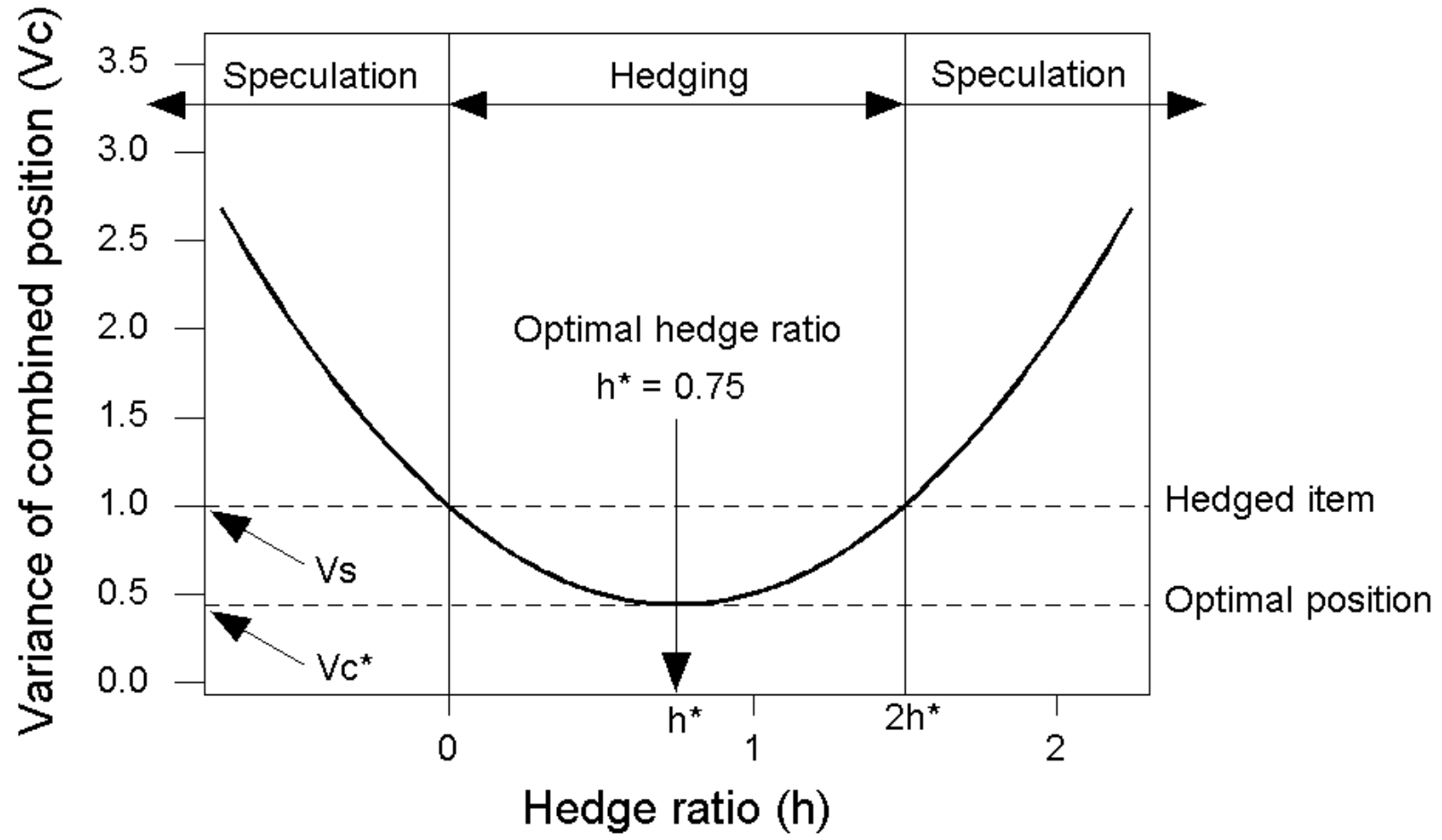

Figure 1: Plot of $V_{C}$ versus $h$ for $\rho=0.75$ when $V_{F}=V_{S}=1.0$ in Expression (1). In this case, $h^{\star}=0.75$, and the entity is considered to be hedging if it chooses any hedge ratio, $h$, such that $0<h<1.5$. The choice of any other value of $h$ constitutes speculation on the part of the entity. 